\title{
Desafíos de la educación superior frente a la pandemia de COVID-19 en América Latina y el Caribe
}

\author{
Dr. Hugo Casanova Cardiel \\ Dr. Pablo Daniel García
}

El gran tema del siglo XXI es hoy el referido a la pandemia de Covid-19. Cada territorio vital, cada ámbito social a lo largo y ancho del mundo, experimenta los efectos de una pandemia que ha desbordado las previsiones de la cosa pública en todos sus niveles y dimensiones, generando un amplio debate en prácticamente cada disciplina académica y científica para elucidar sus riesgos, efectos y alternativas de solución.

Es precisamente en el ámbito del conocimiento especializado donde se ubican las mayores expectativas para la contención y manejo de la calamitosa situación surgida al inicio de la tercera década de este siglo. El conocimiento sobre el virus y su capacidad de contagio, el tratamiento médico de los pacientes afectados, así como el desarrollo de mecanismos de prevención -especialmente vacunas y medios físicos para contener los contagios-, han sido abordados desde el amplio espectro de las ciencias de la salud, la biología y la ingeniería, entre otras.

En particular, desde las ciencias sociales y las humanidades se han generado numerosas investigaciones que apelan a una problemática que se estudia desde las más diferentes disciplinas: jurídicas, sociales, económicas, antropológicas y educativas, entre otras. Es en ese gran campo donde se encuentran adscritas las reflexiones referidas a la educación superior.

En esa área confluyen instituciones diversas y múltiples campos del conocimiento. Se trata de una amplia heterogeneidad institucional, cuyo común denominador está centrado en la formación para la vida profesional y la convivencia ciudadana adulta. Asimismo, es también un campo de estudios dentro del cual se generan investigaciones que atienden a sus más diversos ángulos. Esa es la razón de ser de la revista Educación Superior y Sociedad, y es, precisamente, el sentido de este dossier.

La pandemia en curso ha tenido efectos radicales en la educación superior. El confinamiento social devino en un inédito abandono de las aulas para millones de estu- 
diantes y en la multiplicación de los retos para las instituciones, que se han visto compelidas a ofrecer modalidades emergentes de enseñanza.

El tránsito a dichas modalidades no podía ser terso y, por el contrario, ha estado lleno de retos y dificultades. Así, de una realidad presencial construida y consolidada a lo largo de muchas décadas, se pasó a una situación inexplorada que pone a prueba la solidez de las instituciones.

Un listado incompleto de los ángulos afectados incluiría, entre otros, la discontinuidad curricular y la intrínseca dificultad para trasladar determinadas materias y contenidos desde las aulas hacia los entornos digitales; las dificultades de acceso a infraestructura digital y conectividad por parte de los diversos actores de la educación superior; las limitaciones formativas del profesorado para impartir clases por esa vía y las del alumnado para reenfocar de tal manera sus procesos de aprendizaje; a todo lo cual se suman, por supuesto, los retos planteados en términos del gobierno y la gestión de la educación superior.

Se trata solamente de algunos de los problemas más visibles que se han experimentado en el campo educativo superior a lo largo de ya casi dos años. Pero además, en este apretado recuento de temas críticos no podrían ser omitidas las conocidas asimetrías sociales que se viven en América Latina y el Caribe. La desigualdad e inequidad social de la región han propiciado en muchos casos el abandono y la exclusión de miles de estudiantes, que se han visto en la necesidad de incorporarse al mundo laboral para contribuir al ingreso familiar. Todo ello conforma un escenario de gran complejidad y por momentos, un grave riesgo para el futuro de la educación superior en toda Latinoamérica.

La compilación de artículos que hoy presentamos da cuenta de algunas de las investigaciones que, desde diferentes rincones de la región latinoamericana, se han realizado para echar un poco de luz sobre tanta incertidumbre. $Y$, sin lugar a dudas, pueden ser una herramienta interesante para analizar todos esos hechos y seguir buscando soluciones.

\section{Sobre los artículos que componen el dossier}

En este dossier se abordan algunos de los temas mencionados en los párrafos antecedentes y se ofrecen rutas de análisis -algunas claramente innovadoras- que avanzan en el conocimiento de la educación superior en nuestros países. Se trata de una selección de trabajos que cubre, como sus autores, muy diversas especificidades nacionales, a través de las experiencias de Argentina, Brasil, Cuba, Colombia, Ecuador, 
México, Perú y Venezuela. Hemos resuelto presentarlos en un determinado orden, en función de sus temáticas y enfoques. Un primer grupo de artículos aborda el tema convocante desde una mirada reflexiva y general. Otros analizan el impacto de la pandemia en grupos de instituciones, sea en términos nacionales o subnacionales. Posteriormente presentamos una serie de textos que ponen su énfasis en el impacto de la pandemia en alguna las funciones específicas de la universidad (investigación, extensión, internacionalización). Siguen otros más que presentan la perspectiva de alguno de los actores del nivel superior, en primer lugar los docentes y el impacto en su tarea de enseñanza, pero también la de los estudiantes. Finalmente, completan el dossier artículos que ponen su foco en experiencias pedagógicas específicas.

En la esperanza de que sirva al lector como una brújula orientadora para la lectura, a continuación presentamos una síntesis de esta valiosa compilación de trabajos.

En primer lugar, "Enseñanza superior, pandemia y trabajo: rupturas y continuidades", de Rusvênia Silva y Daniel Santos da Silva. Este artículo se centra en la situación de la educación en Brasil para presentar algunas reflexiones sobre el impacto de la pandemia en la universidad y los desafíos que eso supone, en un momento en que la enfermedad se solapa con el fortalecimiento de los postulados neoliberales del gobierno central, generando un escenario de crisis simultáneas que conviven y se potencian mutuamente. Se revisan allí las drásticas disrupciones que se dan hoy día en las experiencias colectivas de vida en las instituciones del nivel superior y en sus actores, así como los cambios que se avizoran en el futuro cercano.

En segundo lugar, el artículo escrito por Axel Didriksson, Freddy Álvarez, Carmen Caamaño, Damián Del Valle, Daniela Perrotta, Célia Caregnato y Bernardo Miorando, titulado "Universidad y pandemia en América Latina: reflexiones desde la diversidad y la complejidad de un fenómeno en desarrollo". Con una mirada amplia sobre toda la región, se despliegan significativas anotaciones sobre la época de transición que vivieron las universidades ya antes de la llegada de la pandemia, proceso que se ha visto incrementado por la incertidumbre de los últimos meses y que sigue generando interrogantes sobre el devenir futuro. El trabajo compila reflexiones de destacados estudiosos de la educación superior en diversos países de América Latina, en las que se consideran dimensiones fundamentales de las funciones sustantivas de las universidades durante un período, como el de ahora, que plantea la búsqueda de nuevas tendencias de reflexión y debate en el contexto de una crisis generalizada, no sólo sanitaria, sino también educativa, social, económica y mundial.

Sigue"Efectos en los procesos de escolarización superior: un acercamiento al escenario educativo en tiempos de distanciamiento social". Jaqueline Guadalupe Guerrero, José 
Duarte Méndez, Diana Moguel Ruz, José Canto Ramírez y Martín Breton de la Loza ofrecen allí una síntesis de estudios realizados en diferentes países, principalmente latinoamericanos, para señalar las dificultades que docentes, estudiantes y autoridades administrativas han enfrentado al tener que llevar las clases a modalidad virtual. En particular, incursionan en los desafíos que supone el método flipped classroom o de aula invertida, que integra el aprendizaje activo con las TIC. Las autoras y autores realizaron una investigación cualitativa en cuatro universidades, con una muestra de estudiantes de licenciatura y posgrado, y describieron en cada caso la forma en que afrontaron la pandemia y las consecuencias de estar en ambientes virtuales.

"La implementación de iniciativas tecnológicas en las universidades de la Argentina frente al Covid-19", de Giselle González, parte desde una mirada centrada en el territorio para analizar cómo responde la universidad a los desafíos que impone su entorno en el contexto de la pandemia. Toma como objeto empírico algunas iniciativas recientes de vinculación científica y transferencia tecnológica en Argentina y, a partir de un análisis descriptivo que integra fuentes primarias y secundarias, busca mostrar algunas líneas para pensar la innovación en las estrategias de transferencia de tecnologías que favorecen a la comunidad.

A continuación, el artículo de María Fernanda Arias Núñez, Ana María Cambours de Donini y Karina Fabiana Lastra, también con el foco puesto en la situación de las universidades argentinas, se titula "Pandemia y universidades: dificultades y respuestas en el Conurbano Bonaerense". Analiza las consecuencias del coronavirus en universidades ubicadas en la periferia de la Ciudad de Buenos Aires (zona conocida en Argentina como el "conurbano bonaerense"), en tanto que instituciones creadas para paliar las iniquidades en una de las áreas con mayor índice de pobreza en esa nación. Para dar cuenta de las respuestas que desde allí se han dado a la crítica situación generada por el aislamiento social, en particular en contextos de vulnerabilidad, las autoras realizaron entrevistas en profundidad a autoridades de las universidades estudiadas y analizaron documentos gubernamentales y de organismos nacionales e internacionales sobre el tema. En particular, plantean un nuevo rol para esas instituciones, en tanto creadoras de bienes sociales a través de las Secretarías locales de Bienestar y productoras de desarrollos científicos y tecnológicos que responden a las necesidades epidemiológicas del momento.

El sexto aporte a este dossier nos lleva a otro rincón de Latinoamérica: Cuba. "Alternativas en el proceso de formación en las universidades cubanas en etapa de Covid-19", de Osmany Hernández Basulto, Isel Ramírez Berdut y Leandrus Lázaro Hernández Ramírez, analiza las características técnicas, pedagógicas, de diseño y de contenido de 
una muestra de aplicaciones tecnológicas puestas a disposición de los estudiantes universitarios cubanos para desarrollar el proceso de enseñanza-aprendizaje en las condiciones de pandemia.

El siguiente trabajo nos permite explorar la situación del Perú."Impacto de programas formativos orientados al desarrollo competencial de docentes y estudiantes: continuidad de la educación superior en situación de emergencia sanitaria por Covid-19 desde contextos virtuales"fue elaborado por Carme Hernández-Escolano, Yuma Inzolia, David Carabantes, Diego Javier Mendoza, Blanca Bernabé, Mary Elisabeth Morocho e Ivory Mogollón. Analiza un estudio realizado en 13 universidades públicas de esa nación andina, donde UNESCO-IESALC, junto con el gobierno nacional, realizan acciones de cooperación para la mejora de la enseñanza. El trabajo considera las tareas de capacitación dirigidas a docentes, estudiantes y equipo técnico.

Se encuentra luego el trabajo de Niurka Tellez Rodríguez, Víctor Hugo Leyva Sojo y Amanda Estruch Tellez, titulado "Ruta de emergencia en el enfrentamiento a la Covid19 desde la extensión universitaria: constructos y efectos", que expone los constructos teóricos y metodológicos de la ruta de emergencia extensionista seguida por la Universidad de Oriente (Cuba) en el enfrentamiento a la pandemia, así como los factores potenciadores y sus principales resultados. Se sustenta la investigación en una metodología mixta, desde un enfoque dialéctico-materialista, que apela a métodos tan diferentes como el análisis-síntesis, la inducción-deducción y el hermenéutico-dialéctico, sin desdeñar la consulta a especialistas, el análisis documental ni la triangulación de fuentes.

Siguen dos trabajos referidos a la función de la internacionalización. En primer lugar, "Los cambios en las estrategias de internacionalización en las instituciones mexicanas de educación superior a partir de la pandemia por Covid-19", de Magdalena BustosAguirre y Rosa Vega Cano. Aquí las autoras ofrecen los resultados de una investigación cuyo objetivo fue conocer las modificaciones ocurridas en esas estrategias de las IES de México por tal causa. La investigación se llevó a cabo a través de entrevistas al personal encargado de la internacionalización en 25 universidades y sus resultados principales muestran que la estrategia prioritaria era la movilidad estudiantil, la cual fue sustituida por otras formas de movilidad y docencia, como la internacionalización en casa y la participación en proyectos internacionales de cooperación académica, hecho que da cuenta, además, de los matices que la transición ha tenido en las diferentes instituciones.

También referido a los procesos de internacionalización, compartimos el artículo titulado "La movilidad académica internacional ante la pandemia del Covid-19: una pri- 
mera aproximación", de José Antonio Quinteiro Goris. Se trata de un análisis del fenómeno de la movilidad académica internacional y de los probables impactos, en lo inmediato y a corto plazo, que tendrá en ella la pandemia. El texto brinda significativas pinceladas de la movilidad académica internacional en América Latina, particularmente en torno a sus principales polos de atracción actual, y plantea algunos probables impactos, a partir del análisis e interpretación de la información recogida como resultado de una revisión documental.

A continuación sigue una serie de artículos que ponen especial atención en los docentes/investigadores que se desempeñan en las universidades de la región. Es el caso de "La regulación de la educación a distancia y la experiencia previa de los académicos argentinos para desarrollar su actividad docente en entornos virtuales antes de la irrupción de la pandemia", presentado por María Catalina Nosiglia y Brian Fuksman. Se proponen ambos caracterizar la experiencia de los académicos argentinos, previa a la irrupción de la pandemia en marzo del año 2020, en el desarrollo de su actividad docente en entornos virtuales y en la evolución normativa de la educación a distancia en Argentina. El diseño metodológico implicó la aplicación de una encuesta a una muestra representativa de académicos de las universidades nacionales argentinas, en el marco del proyecto internacional APIKS (Academic Profession in the Knowledge-Based Society). Así también, con un foco de análisis puesto en los docentes, pero en este caso de Colombia, sigue el artículo titulado "Percepciones de docentes universitarios frente al cambio de modalidad presencial a remota por el Covid- 19: comparación entre profesores presenciales y virtuales", elaborado por John Jairo Briceño Martínez y Martha Patricia Castellanos Saavedra. En este caso se trata de una investigación de corte exploratorio que involucró a 749 docentes universitarios de la Fundación Universitaria del Área Andina (Colombia), a los que se les administró un instrumento para dar cuenta de dificultades y necesidades de estudiantes y docentes que, debido a la pandemia, tuvieron que migrar a una modalidad de educación virtual.

"Enseñanza a distancia y formación continua en servicio en posgrados, stricto sensu", de Fabrício de Andrade y Dorotea Frank Kersch, , se construye en torno a un estudio de historia profesional y tiene como objetivo analizar los aprendizajes de los docentes que se capacitan para el diseño de cursos híbridos y multimodales, con inclusión de tecnologías digitales. El trabajo narra la historia de Martina, una docente participante de estas instancias de formación, y apela a su caso para poner en reflexión y discusión los aprendizajes de esta época.

A continuación viene el trabajo de Paula Seminara Torcivia, titulado "De los efectos de la pandemia de Covid-19 sobre la deserción universitaria: desgaste docente y bie- 
nestar psicológico estudiantil". Con foco en la situación de Argentina, se propone mostrar las principales dificultades y posibles ventajas -para estudiantes y docentessurgidas a raíz de la necesidad de continuar el curso didáctico en modalidad virtual. Dentro de los factores indagados, enfatiza en el bienestar psicológico y el desgaste docente. Para ello aplicó una encuesta dirigida a ambos grupos, pertenecientes a la carrera de Bioingeniería en la Universidad Nacional de San Juan, Argentina.

"Educación remota de emergencia: experiencias de estudiantes de una Unidad Académica Regional de la BUAP durante la pandemia de Covid-19" es el décimo quinto trabajo y fue producido por Abelardo Romero Fernández, Laura Villanueva Méndez, Ana Liviere Vargas Vizuet y Fabio Morandín Ahuerma. Presenta los resultados de una investigación cuantitativa de alcance exploratorio, que tuvo por objetivo recuperar y explorar las experiencias de los estudiantes de nivel licenciatura que tuvieron que concluir el semestre de la primavera mexicana de 2020 por medio de clases no presenciales, con los medios de que disponían durante la etapa de mayor incertidumbre en la expansión de la enfermedad. Para realizar el trabajo de campo se aplicó un cuestionario, con preguntas cerradas y abiertas, a 124 estudiantes de carreras del área de salud en el estado de Puebla.

También con foco en el impacto de la pandemia en las trayectorias de formación de estudiantes universitarios, el artículo siguiente se titula "Educación superior en el Perú en tiempos de pandemia y sus efectos en las trayectorias de estudiantes universitarios indígenas", y lo suscriben Inés Olivera Rodríguez, Alejandro Saldarriaga y María Amalia Pesantes, de ese país andino. Allí ofrecen un análisis documental y testimonial acerca de cómo la pandemia ha afectado de manera particular a las y los indígenas, así como en torno a las deficiencias de las estrategias adoptadas por el Estado peruano para que el estudiantado indígena pueda continuar con sus estudios durante la crisis sanitaria. Sigue "La pandemia como factor de exclusión de los estudiantes con discapacidad en la educación superior", de Judith Pérez-Castro. La autora describe las dificultades que han enfrentado en México tanto estudiantes como académicos e investigadores frente a las actividades a distancia y debido a la cancelación de proyectos, entre otras causas. En particular, analiza los efectos que han tenido las restricciones por motivo de salud pública entre los estudiantes mexicanos con alguna discapacidad, al tiempo que resalta las carencias de todas las universidades para brindar una educación en línea a esas personas, tan vulnerables como el estudiantado de bajos recursos, las mujeres y la población de origen indígena.

También el artículo que sigue hace foco en la situación de los estudiantes con discapacidad. "Universitarios con discapacidad: realidades y desafíos en contexto de 
pandemia de la Red Interuniversitaria Latinoamericana y del Caribe sobre Discapacidad y Derechos Humanos", de Arlett Krause, Georgina García, Sandra Katz y Susana Rodríguez, busca contextualizar a los países latinoamericanos en los marcos de la discapacidad, la educación superior y el Covid-19. La información que presenta fue provista por los referentes en cada país de la mencionada Red, con el objetivo de describir las realidades nacionales en esa circunstancia y las respuestas de los gobiernos y universidades para garantizar accesibilidad académica y calidad de educación a ese alumnado. Abarca todo un conglomerado de países: Argentina, Bolivia, Brasil, Chile, Colombia, Costa Rica, Ecuador, Guatemala, México, Panamá, Paraguay, Perú, Uruguay y Venezuela.

A continuación, sigue una serie de artículos que analizan experiencias específicas y valiosas. Se incluye así el titulado "Educación remota de emergencia en la formación inicial docente: desafíos y posibilidades en el contexto de la pandemia de civod-19", de Viviane Potenza Guimarães Pinheiro y Beattriz Guedes de Seixas. Centrado en la formación docente en Brasil, analiza la migración a la educación remota, bajo parámetros de emergencia, de una disciplina previamente planificada para el modelo presencial en una universidad pública brasileña, tema que aborda a través de entrevistas a 16 estudiantes que participan en el curso. El análisis cualitativo y descriptivo de las percepciones y sentimientos de los alumnos marcó los desafíos y las posibilidades de tal experiencia.

El vigésimo artículo se titula "Políticas académicas en la emergencia socio-sanitaria por Covid-19: una experiencia de aprendizaje institucional". Viviana Macchiarola y Daiana Anahí Bustos, sus autoras, presentan los resultados de una investigación cualitativa realizada en la Universidad Nacional de Río Cuarto (UNRC), en Argentina, cuyas autoridades académicas comentaron cómo, para hacer frente a la situación determinada por la pandemia, las decisiones se tomaron pragmáticamente y se llegó a acuerdos y consensos con todos los actores involucrados.

El siguiente en el dossier se titula "Relato de experiência da gestão democrática da Universidade Estadual do Rio Grande do Sul em tempos de pandemia". Elaborado por Caroline Tavares de Souza Clesar, Rochele da Silva Santaiana, Débora Vom Endy Percila Silveira de Almeida, reporta la experiencia de la Universidad Estadual de Rio Grande do Sul en el retorno gradual y no presencial a las actividades docentes de esa universidad durante la pandemia. Se caracteriza por retratar una experiencia de investigación empírica con estudiantes, profesores y personal técnico de la institución, con el fin de brindar datos sobre las condiciones de acceso a herramientas tecnológicas, internet y mapeo de grupos de riesgo. 
Finalmente, cierra el dossier el artículo titulado “La educación virtual en la pandemia por el SARS-CoV-2: desafío para los docentes", de Pascual Valdez, Susana Elsa Salomón, Alejandro Cragno, Hugo Fernán Milione, Dario Leff, Julio Guillermo Wacker, Marcelo Yorio y Alejandra Gaydou. Este trabajo explora procesos y metodologías docentes durante la pandemia y los espacios de enseñanza-aprendizaje-evaluación en Perú. Se analiza la educación virtual, a distancia, y la educación remota de emergencia, para lo cual se realizó una encuesta en línea, estructurada, autoadministrada y anónima. El trabajo busca dar pistas de la mutación vivida en la educación superior peruana, a fin de contribuir a la toma de decisiones que permitirán planificar mejores estrategias para facilitar el mejor uso de las nuevas tecnologías y la virtualidad.

Para cerrar, quisiéramos agradecer a todas y todos los autores que han aportado su trabajo para la construcción de este número. Además, al UNESCO-IESALC y al equipo editorial de la revista Educación Superior y Sociedad, especialmente a Débora Ramos, por permitirnos contribuir -desde este amplio dossier - a las discusiones que se desarrollan en el campo de la educación superior contemporánea. También, por supuesto, al nutrido equipo de dictaminadores que desde diversos ámbitos regionales se sumaron al esfuerzo común de construir un conjunto de trabajos consistente en términos académicos.

Hoy, con alegría, vemos como en muchos países del mundo la pandemia parece retroceder y poco a poco vamos retomando nuestras actividades cotidianas con cierta "normalidad". Las universidades de nuestra región, aunque lentamente, van llenándose de vida y nuevos desafíos aparecen. Es tiempo de balance y planificación, tiempo de reflexionar y evaluar sobre lo vivido. Estamos convencidos de que la investigación educativa puede aportarnos importantes pistas para pensar el futuro de nuestras instituciones. Deseamos que esta época, que tanto dolor ha traído, también sea fuente de innovaciones y aprendizajes para pensar sistemas universitarios más justos e incluyentes, donde el derecho a la educación superior se concrete para cada vez más habitantes de nuestra región. 


\section{BIONOTAS}

Hugo Casanova Cardiel. Doctor en Filosofía y Ciencias de la Educación por la Universidad de Barcelona, España. Investigador titular y director del Instituto de Investigaciones sobre la Universidad y la Educación, así como profesor en el programa de posgrado en Pedagogía de la Universidad Nacional Autónoma de México, UNAM. Sus líneas de investigación son múltiples: a) política y gobierno de la universidad; b) historia contemporánea de la UNAM; $y$ c) política educativa en México. Ha publicado 12 libros como autor o editor y más de 80 artículos y capítulos de libro. Ha impartido clases y conferencias en universidades de México y el extranjero, lo que incluye la Universidad de Texas (Austin, EE.UU.) por un año y la Universidad de Barcelona (España) por dos años. Es integrante del Sistema Nacional de Investigadores en el nivel III y forma parte de la Academia Mexicana de Ciencias, así como de la Sociedad Española de Pedagogía y del Consejo Mexicano de Investigación Educativa, del que fue presidente en el bienio 2010-2011.

Correo electrónico: hcc2010@gmail.com

Pablo Daniel García. Es doctor en Educación (UNTREF-UNLA), magister en Políticas y Administración de la Educación (UNTREF), especialista en Gestión y Conducción del Sistema Educativo y sus Instituciones (FLACSO) y en Métodos y Técnicas de Investigación Social (CLACSO), además de profesor y licenciado en Ciencias de la Educación (UBA). También, profesor-investigador en UNTREF, coordinador del Programa de Posgrados en Políticas y Administración de la Educación (UNTREF) y coordinador académico del Doctorado en Política y Gestión de la Educación Superior (UNTREF). Adicionalmente, es secretario editorial de la Revista Latinoamericana de Políticas y Administración de la Educación (RELAPAE), del NIFEDE-UNTREF, editor asociado en la revista Educación Superior y Sociedad (UNESCO-IESALC), consejero de la Sección Política Educativa de LASA (Latin American Studies Association) y forma parte de la comisión directiva de la Sociedad Argentina de Estudios Comparados en Educación (SAECE).

Correo electrónico: pgarcia@untref.edu.ar 